P118 (continued)

frequency at Time 2. Females in this group also reported higher frequencies of eating breakfast, eating a staple dish at breakfast, and eating a vegetable dish at breakfast.

Conclusions and Implications: This study implies that frequent family breakfast during adolescence is associated with better breakfast diet at young adulthood among females.

Funding: Ministry of Education, Culture, Sports, Science and Technology, Japan

\section{P119 Managing Youth Recreational Sports to Promote Healthier Eating}

Andrew Ramsey, University of North Carolina, Greensboro; Anthony Weaver, PhD, Elon University; Lauren Haldeman, PhD, lahaldem@uncg.edu, University of North Carolina at Greensboro, 1000 Spring Garden Street, Greensboro, NC 27402

Objective: To examine snacking and eating behaviors among youth recreation participants.

Study Design, Setting, Participants: Youth recreational playing fields in central North Carolina. English speaking parents of participating children.

Outcome Measures and Analysis: Descriptive analysis of survey responses to four categories: importance of snacks provided at youth recreation events, reason for snack choice, snacks purchased most often, and family eating behaviors on game/practice nights.

Results: Parents $(\mathrm{N}=200)$ of primarily white males aged 8 years responded to the survey. More than half (56.5\%) of the children play recreational soccer. Most parents (67.5\%) reported that snacks are important at games. Taste $(56 \%)$ and convenience $(62 \%)$ were the two most important factors when deciding the type of snack to bring. Snacks provided mostly included: various chip types and juice boxes. About half of respondents reported that family eating behaviors change on games/practice nights with $62 \%$ reporting going out to eat.

Conclusions and Implications: Youth recreation provides an opportunity for children to participate in regular physical activity and promote good health; yet parents in this study report largely providing high fat, high sugar team snacks. Additionally, families often eat out on game/practice nights. Education should be provided to parents regarding the purchase of healthy snacks and strategies for maintaining healthy eating behaviors on game/practice nights. Nutrition educators should also work with departments of parks and recreation and team coaches to plan for ways to provide healthy snacks to their team participants. Policy changes regarding the management of game/practice schedules and team snack offering may help to better support healthy eating among youth recreation families.

Funding: None

\section{P120 Meeting the Added Sugars Guideline: Impact on Whole Grains, Vegetables, Fruit, and Dairy Intakes of Children}

Shanthy Bowman,PhD, shanthy.bowman@ars.usda.gov, US Department of Agriculture, 10300 Baltimore Avenue, BARC-West, Beltsville, MD 20705

Objective: The 2015-2020 Dietary Guidelines for Americans recommend that added sugars intake not exceed 10 percent of total energy intake. The research compares the mean energy, whole grains, vegetables, fruit, and dairy intakes of children 2-19 years, grouped based on whether they met the added sugars guideline or not.

Study Design, Setting and Participants: What We Eat in America, National Health and Nutrition Examination Survey 2011-12, day 1 dietary data were used for the study. The study included 3132 children.

Outcome Measures and Analysis: Mean intake estimates of the two groups were compared; and a p-value less than 0.01 was considered significantly different.

Results: About 27 percent of them met the added sugars guideline. These children had significantly higher intakes of vegetables $(1.1 \pm 0.06$ vs. $0.8 \pm 0.03$ cup eq.), fruit ( $1.4 \pm 0.06$ vs. $1.0 \pm 0.07$ cup eq.), and dairy ( $2.5 \pm 0.11$ vs. $2.2 \pm 0.08$ cup eq.) than the children who consumed more than 10 percent of calories from added sugars. No significant differences were noted in the whole grains $(0.73 \pm 0.03$ oz. eq.) and energy (1983 \pm 23 calories) intakes between the two groups. About 23 percent of non-Hispanic white, 25 percent of non-Hispanic black, 40 percent of non-Hispanic Asian, and 31 percent of Hispanic children met the added sugars guideline.

Conclusions and Implications: The study showed children not meeting the added sugars guideline had lower intake of nutritious foods. Dietary interventions aimed at children to increase their fruit, vegetables, whole grains, and dairy intake should simultaneously address choosing foods with little or no added sugars. This study findings are useful to nutrition educators, dieticians, and nutritionists working with children and their parents.

Funding: USDA

\section{P121 Mixed Methods Evaluation of Growing Healthy Kids School Enrichment Kit Program}

Elisha Hall, PhD, RD, LD, elisha@huskers.unl.edu, Feeding Texas, 1524 South I H 35 \#342, Austin, TX 78704; Paula K. Ritter-Gooder, $P h D, R D$, University of NebraskaLincoln; Alyssa Havolvic, MS, RD; Mindy AndersonKnott, MA; Alian Kasabian, PhD; Weiwen Chai, PhD; Julie Albrecht, PhD, RD

Objective: To evaluate the Growing Healthy Kids School Enrichment Kit Program (SEKP).

Study Design, Setting and Participants, and Intervention: The SEKP is a Social Cognitive Theory-based, 